\title{
The Effects of Finite Length on the Electronic Structure of Carbon Nanotubes
}

\author{
Alain Rochefortt \\ Centre de Recherche en Calcul Appliqué (CERCA), 5160 boul. Décarie, bureau 400, Montréal, (Québec) Canada H3X 2H9 \\ Dennis R. Salahubl \\ Département de Chimie, Université de Montréal, C.P. 6128, Succ. Centre-Ville, Montréal, (Québec) Canada H3C 3J7 \\ Centre de Recherche en Calcul Appliqué (CERCA), 5160 boul. Décarie, bureau 400, Montréal, (Québec) Canada H3X $2 H 9$ \\ Phaedon Avourisit \\ IBM Research Division, T.J. Watson Research Center, P.O. Box 218, Yorktown Heights, NY 10598, USA
}

(October 15, 2018)

\begin{abstract}
The electronic structure of finite-length armchair carbon nanotubes has been studied using several ab-initio and semi-empirical quantum computational techniques. The additional confinement of the electrons along the tube axis leads to the opening of a band-gap in short armchair tubes. The value of the band-gap decreases with increasing tube length, however, the decrease is not monotonic but shows a well defined oscillation in short tubes. This oscillation can be explained in terms of periodic changes in the bonding characteristics of the HOMO and LUMO orbitals of the tubes. Finite size graphene sheets are also found to have a finite band-gap, but no clear oscillation is observed. As the length of the tube increases the density of states (DOS) spectrum evolves from that characteristic of a zero-dimensional (0-D) system to that characteristic of a delocalized one-dimensional (1-D) system. This transformation appears to be complete already for tubes 5-10 nm long. The chemical stability of the nanotubes, expressed by the binding energy of a carbon atom, increases in a similar manner.
\end{abstract}

\section{INTRODUCTION}

Carbon nanotubes are a new form of carbon with rather unique properties] 3 . A single wall nanotube can be considered as resulting from rolling up a single graphene sheet to form a hollow cylinder. The chirality and diameter of a particular tube can be described in terms of a role-up vector $C=n \vec{a}+m \vec{b} \equiv(n, m)$, where $\vec{a}$ and $\vec{b}$ denote the unit vectors of the hexagonal honeycomb lattice, and $\mathrm{n}$ and $\mathrm{m}$ are integers $\mathrm{B}$. In a 2 -D graphene sheet the $\pi$-bonding and $\pi^{*}$-antibonding states become degenerate at the K-point of the hexagonal Brillouin zone, resulting in a zero band-gap semiconductor. In a nanotube, quantization of the wavefunction along its circumference restricts the allowed wavevectors to certain directions of the graphite Brillouin zone so that $C \cdot k=2 \pi j$, where $j$ is an integer. If at least one of these wavevectors passes through the $\mathrm{K}$ point, the tube is metallic, otherwise it is a semiconductor with a finite band-gap. Thus, $(n, 0)$ zig-zag tubes are expected to be metallic if $n / 3$ is an integer, and semiconducting otherwise 6 . 
As the role-up vector $C$ rotates away from the $(n, 0)$ direction, the resulting $(n, m)$ tubes are chiral and are expected to be metallic if $(2 n+m) / 3$ is an integer. Otherwise they are semiconductors with a gap $\propto 1 / R$, where $R$ is the tube radius. Finally, when $C$ is rotated $30^{\circ}$ away from the $(n, 0)$ direction, $n=m$ and the resulting armchair tubes are metallic. Recent scanning tunneling microscope studies have verified the basic conclusions of simple theory

The above predictions assume a semi-infinitely long tube, and corresponding experimental electronic structure studies have utilized tubes several microns in length. It is very important to know how an additional confinement of the electrons, along the nanotube axis, in finite length tubes will affect the tube's electronic structure. As the tube length is diminished, there should be a transition from a 1-D structure (quantum wire) to a 0-D structure (quantum dot). Currently, little is known about the effects of finite length on the properties of carbon nanotubes. Recently, however, Venema et al. 2 have been able to cut nanotubes into segments of a few tens of nanometers length and, using tunneling spectroscopy, obtained evidence of increased band-gaps in these short tubes. Understanding quantum size effects in carbon nanotubes, besides the basic scientific value of understanding a prototypical 1-D to 0-D transition, is essential in device applications of nanotubes. The miniaturization offered by nanotube-based devices requires that not only their diameter but also their length should be in the nanometer range. For example, micrometer-long nanotubes have been used as Coulomb islands in single electron transistors (SET) operating at liquid helium temperatures 10.11 .10 . By using shorter tubes, the operating temperature of SETs can be raised drastically. In general, the electrical properties of nanotubes may be tailored by selecting their length.

To obtain the needed insight into the electronic structure of short nanotubes and learn about their stability (information useful in studies of growth mechanisms) we performed electronic structure calculations on different length $(6,6)$ armchair nanotubes. To determine the local properties and the spatially-resolved electronic structure of finite-size nanotubes, quantum-chemical methods are more suitable than band-structure calculations. However, the theoretical determination of electronic properties, such as the band-gap value, remains a difficult task, as has been shown in studies of low band-gap polymers12. To compensate for the weaknesses of the various quantum chemical techniques, we utilized a number of both first-principles (density functional and Hartree-Fock) and semi-empirical computational techniques (MNDO-PM3 and extended Hückel). We used the above techniques to calculate the band-gap values, the DOS spectrum and the binding energy of carbon atoms as a function of the length of the tube segment. While, according to the discussion in the Introduction, infinitely long $(6,6)$ nanotubes should be metallic, all computational techniques predict a band-gap for short nanotubes whose value decreases towards zero with increasing tube length. Interestingly, the variation of the band-gap is not monotonic but shows strong oscillations as a function of tube length. While all computational techniques used predict these oscillations, the actual values of the band-gap vary strongly with the technique used. Computed DOS spectra and local density of states distributions show that the transition from a 0-D to a 1-D-like spectrum takes place already in very short 5-10 nm tubes. The binding energy of the carbon atoms increases with increasing tube length in a similar manner. The behavior of finite graphene sheets is also investigated. 


\section{COMPUTATIONAL DETAILS}

Electronic structure calculations were performed using both ab-initio: Hartree-Fock (HF) and Density Functional Theory (DFT), and semi empirical: Modified Neglect of Differential Overlap - Parametrized Model 3 (MNDO-PM3) and extended Hückel (EHMO) computational methods. In the HF calculations, we employed a parallel version of the GAMESS program 13 in which STO-3G quality basis sets were employed for carbon and hydrogen. For the DFT

computations, we used the deMon-KS software14 16. The calculations were performed with Huzinaga's minimal basis sets in conjunction with the generalized gradient approximation (GGA) of Perdew and Wang for exchange 18 and correlation 19 . The semi-empirical calculations were carried out with the MNDO-PM3 method 20,21 included in the GAMESS program13, while for the EHMO calculations we used the program included in the YAeHMOP package 22.

In the carbon nanotube models, the $\mathrm{C}-\mathrm{C}$ and $\mathrm{C}-\mathrm{H}$ bond length were fixed at the values observed in bulk graphite 23 which are 1.42 and $1.09 \AA$, respectively. All calculations were performed at fixed geometries without optimization of the atomic structures. The elementary unit used, called here a section, is defined as a single circular plane of carbon atoms that are packed along the length of the nanotube (note: the distance between two consecutive sections in the nanotube is $1.22 \AA$ ). The dangling bonds at the ends of the tube were saturated with hydrogen atoms. The energy band-gap is obtained as the difference between the LUMO and HOMO energies, while the Fermi energy $\left(E_{F}=0\right.$ eV $)$ is the mean energy between LUMO and HOMO.

Density of states (DOS) plots were generated by convoluting the computed electronic structure with a 50:50 combination of Gaussian and Lorentzian functions. In order to analyze the nature of the energy bands, we performed a series of projections to obtain the local density of states (LDOS), where each molecular orbital was weighted by the contribution obtained from a Mulliken analysis of specific carbon atoms. The general expression used to generate the DOS plots is :

$$
\operatorname{DOS}(\epsilon)=\sum_{i=1}^{N}\left(\left[\frac{n_{i}}{\omega\left(\frac{\pi}{2}\right)^{1 / 2}} \cdot \exp \left(\frac{-2\left(\epsilon-\epsilon_{i}\right)^{2}}{\omega^{2}}\right)\right]+\left[\frac{2 n_{i} \omega}{\pi\left(\omega^{2}+4\left(\epsilon-\epsilon_{i}\right)^{2}\right)}\right]\right)
$$

where $\omega$ is the resolution, $n_{i}$ is the population of the level $i$ and $\left(\epsilon-\epsilon_{i}\right)$ is the energy difference between the energy $\epsilon$ and the eigenvalue $\epsilon_{i}$.

\section{RESULTS AND DISCUSSION}

To evaluate the influence of finite length on the electronic properties of the nanotubes, we calculated the electronic structure of different length segments of a $(6,6)$ nanotube. As Figure 1 shows, all computational techniques used predict that, unlike the infinite tube that is metallic, very short $(<100 \AA)$ nanotubes have an energy band-gap $\left(E_{g}\right)$. Furthermore, Figure 1 reveals that $E_{g}$ shows a regular oscillatory dependence on tube length. The oscillation of 
the band-gap values is particularly strong in the HF method where $E_{g}$ varies from 2.8 to $9.0 \mathrm{eV}$ for tubes shorter than $20 \AA$. The semi-empirical MNDO-PM3 method gives results comparable to those obtained by HF. On the other hand, extended Hückel molecular orbital (EHMO) and density functional theory - generalized gradient approximation (DFT-GGA) methods lead to quite similar values of the band-gap. The oscillation amplitude decreases, and the band-gap value converges slowly to zero for larger nanotubes. For a $96 \AA$ long nanotube, EHMO predicts a metallic behavior while MNDO-PM3 still shows a band-gap of $2.0 \mathrm{eV}$. The comparison of the different quantum-chemical methods used to determine $E_{g}$ will be discussed in more detail below.

The inset in Figure 1 gives an expanded view of the band-gap oscillations for nanotubes shorter than $25 \AA$. All computational methods predict a regular and converging oscillation of $E_{g}$. The oscillation is related to the structure of the nanotube; high band-gap values are invariably obtained for tubes having $(3 n+1)$ (where $n=1,2, \ldots)$ sections (reminder: a section contains a single circular plane of carbon atoms). Furthermore, consecutive medium or low band-gap values are periodically observed for nanotubes having $(3 n)$ and $(3 n-1)$ sections. The main difference between the oscillatory behavior of $E_{g}$ obtained with the different computational methods lies in the different length needed for an inversion between medium and low band-gap behavior to occur. The different oscillations observed, as well as the low to medium band-gap inversion for small nanotubes can be explained in terms of the changing bonding characteristics of the HOMO and LUMO orbitals.

Figure 2 shows the nodal structure of the frontier $\pi$-orbitals for the nanotube models, while Figure 3 gives the energies of ten molecular orbitals (obtained from EHMO theory) which lie near the band-gap region. The relative stability of frontier orbitals (HOMO and LUMO) for the first three models (S2, S3 and S4 with respectively, 2,3 and 4 sections) is based on the nature of the bonding along the circumference of the tube, i.e intra-section interactions, as well as the bonding along the tube axis, i.e. inter-section interactions. Furthermore, the S2, S3 and S4 models constitute the repeat units from which the HOMO/LUMO orbitals of the longer tubes may be constructed. From Figure 2, we see that the HOMO for S2 (two section model), the first member of the $\mathrm{S}(3 n-1)$ group, has bonding character along the circumference, while the LUMO has bonding character between sections, i.e., along the tube axis. We should then expect the energy difference between HOMO and LUMO of the S2 structure to be small, leading to a low band-gap. On going from the $\mathrm{S} 3(\mathrm{~S}(3 n)$ group) to the $\mathrm{S} 4(\mathrm{~S}(3 n+1)$ group) structures, we observe from Figure 2 that the HOMO acquires an additional bonding character along the tube axis and hence is stabilized more. On the other hand, the LUMOs of both S3 and S4 type structures show antibonding inter-section interactions which lead to a larger band-gap. The gap is lower for S3 due to the net bonding character of the LUMO along the circumference. The structure and the stability of the frontier orbitals for longer nanotubes are directly related to the characteristics of those three first structures. The $\mathrm{S}(3 n-1)$ group (i.e. S2, S5, S8, . ) is characterized by non-bonding inter-section interactions, as is evident by considering the orbital nodal properties in Figure 2. The stability of the frontier orbitals in the $\mathrm{S}(3 n-1)$ group is weakly influenced by inter-section interactions. The slight destabilization of the frontier orbitals is due to long-range antibonding lateral interactions that reduce slowly the band-gap with increasing tube 
length. In the $\mathrm{S}(3 n)$ group (S3, S6, S9, . ) , the decreasing band-gap is caused by a destabilizing lateral antibonding interaction in the HOMO coupled with a stabilizing lateral interaction in the LUMO. The lateral interactions have a strong influence on the value of the band-gap of the $\mathrm{S}(3 n)$ group so that it actually becomes lower than the band-gap value of the $\mathrm{S}(3 n-1)$ group at $n=3$. This behavior occurs at different tube lengths depending on the computational method used to determine the energies of the molecular orbitals. Finally, the $\mathrm{S}(3 n+1)$ group that is characterized by high band-gaps follows a similar trend as the $\mathrm{S}(3 n)$ group with increasing nanotube length; a destabilization of the HOMO through antibonding interactions between sections and stabilization of the LUMO through partly bonding lateral interactions.

Band-gap oscillations were also reported recently by Yoshizawa et al 24 for 2-D polyphenanthrenes on the basis of extended Hückel band structure calculations. Since, instead of the carbon section used here, a phenanthrene-edge structure was used as the elementary building unit in that study, high band-gaps were found in systems with $(6 n-2)$ sections rather than $(3 n+1)$ sections. Nevertheless, although the compositions of the frontier orbitals of the $(6,6)$ armchair nanotube are to a certain extent different from those of the periodic 2-D polyphenanthrenes, both structures give rise to a similar pattern of band-gap oscillations. In Figure 4 we compare the variation of the band-gap value for a perfect $(6,6)$ nanotube to that of an equivalent 2-D graphene fragment (whose broken C-C bonds were saturated with hydrogen atoms), and that of the periodic sheet of 2-D polyphenanthrenes as determined by Yoshizawa24. We find that the growth of the finite 2-D graphene sheet does not lead to a clear band-gap oscillation pattern and the $E_{g}$ rapidly converges to low values. Furthermore, the finite graphene sheet gives quite different $E_{g}$ values than the infinite polyphenanthrene sheet. Figure 5 shows the influence of the nanotube diameter on the magnitude of the band-gap oscillations with respect to the fluctuations observed for a periodic graphene sheet. Increasing the nanotube diameter by going from the $(6,6)$ to the $(10,10)$ tube, brings its band-gap value closer to that of the periodic 2-D graphene sheet. However, the $1 /$ (diameter) scaling of $E_{g}$ predicted for infinite tubes阳 is not observed in very short tube segments.

Crucial to the understanding of the nanotube growth process are changes in the binding energy of $\mathrm{C}$ atoms as a function of the increasing nanotube length. Figure 6 compares the binding energy (BE) of carbon atoms in a finite $(6,6)$ nanotube as determined by different computational techniques. A converged BE value indicates that all carbon atoms are nearly equivalent to each other. Major changes are observed only for very short tubes where the BE found to increase sharply up to about $15 \AA$, and then changes more gradually upto $96 \AA$ (not shown). The smaller changes in $\mathrm{BE}$ observed with the EHMO method can be understood as due to the treatment of long-range interaction in EHMO theory; carbon atoms are weakly perturbed by second and higher neighbor carbon atoms. The highest estimate of BE is obtained with MNDO-PM3 (7.0 eV / C atom), and the lowest with EHMO (5.5 eV / C atom). The BE obtained with the ab-initio DFT-GGA (estimated) and HF lie between these two extremes. The low binding energy obtained for short tubes suggests that carbon in short nanotubes would be more reactive. 
Although the energetic properties of nanotubes seem to converge rapidly, a sufficiently long nanotube model is necessary to reproduce the main characteristics of the electronic structure of a quasi-1D periodic system. The influence of the length on the general electronic structure of a $(6,6)$ armchair nanotube is reported in Figure 7 obtained using the EHMO method. The nanotube electronic structure is characterized in terms of the position and the intensity of the main DOS peaks, the magnitude of DOS at the Fermi level, and the presence of fine structure. The spectrum of the very short $4 \AA$ ( 4 carbon sections) model is characteristic of a 0 -D (quantum dot) system with discrete quantum levels. However, the band position and its overall DOS profile is not drastically different from those of more extended systems. The DOS profile for a $96 \AA$ long model, on the other hand, clearly reveals the presence of characteristic fine structures at high binding energy (BE) between -22 and -12 eV. These structures are "van Hove singularities" characteristic of periodical one-dimensional (1-D) system 25. Each of these peaks is characterized by a specific number of nodes in the wavefunction along the circumference of a single nanotube section, while the $1 / \sqrt{E-E_{0}}$ tail reflects the free electron character along the tube axis. In fact, these singularities are becoming evident even in the $47 \AA$ nanotube that represents the minimum length nanotube that exemplifies such 1-D-like characteristics. For nanotubes larger than $47 \AA$, the main changes in electronic structure occur around the Fermi level, where the DOS is found to increase slowly with increasing nanotube length. Although it gives a different estimate for the band-gap, the semi-empirical MNDO-PM3 method shows similar trends as EHMO concerning the electronic structure of the growing nanotubes.

Figure 8 gives a more detailed description of the electronic structure of the $96 \AA$ nanotube 26 . In the general DOS diagram, the van Hove singularities at high $\mathrm{BE}$ are $\sigma$-states that originate from $\mathrm{C}(2 s)$ atomic orbital combinations. The $\sigma$-states formed from $\mathrm{C}(2 p)$ orbitals lie at lower $\mathrm{BE}$ between -11 and $-4 \mathrm{eV}$, while $\pi$-states extend from -5 to 0 $\mathrm{eV}$. These states are important as they are responsible for the electrical properties of nanotubes. The states above the Fermi level are mainly $\pi^{*}$-states, while $\sigma^{*}$-states extend above $10 \mathrm{eV}$. The inset gives an expanded view of the fine structure of the $96 \AA$ nanotube near the Fermi energy. In addition, the values of the local density of states (LDOS) near the Fermi energy for sections ranging from the tube boundary (index 1) to the middle of the tube (section 40) are plotted. To construct these LDOS plots, we summed over the density of the twelve carbon atoms contained in that section of the nanotube. From figure 8 it can be seen that the LDOS of the $\pi$-states near $E_{F}$ hardly changes along the length of the tube, indicating that these states are indeed well delocalized within this short tube.

Finally, we shall compare briefly the results obtained with the different computational techniques. As shown in Figure 1, both HF and MNDO-PM3 methods give a large band-gap (9.0 and $6.5 \mathrm{eV}$, respectively) for a short 4 $\AA$ nanotube, while DFT and EHMO give moderate band-gap values (2.4 and $1.8 \mathrm{eV}$, respectively). Due to the poor description of unoccupied orbital energy levels in Hartree-Fock theory, the band-gap values are overestimated by several electron volts for low band-gap polymers27 29. The problem is slightly less important in the MNDOPM3 method, where the parametrization includes a treatment of correlation through the fitting with experimental data30 32. Previous MNDO-band structure calculations have predicted a metallic behavior $\left(E_{g}=0 \mathrm{eV}\right)$ for periodic 2-D graphite 33 34. This suggests that the computed band-gap should converge to zero for extended nanotube models; 
we estimate from Figure 1 that nanotubes larger than $450 \AA$ would be metallic. On the other hand, DFT-based methods tend to systematically underestimate the band-gap value of low band-gap polymers3. However, recent developments of DFT/hybrid functionals that include a weighted contribution of Hartree-Fock and DFT exchange allow a better evaluation of the band-gap value2.36. Given that the computed band-gaps with EHMO are similar to the DFT results, we may conclude that the band-gap values are also underestimated with EHMO. Based on DFT and EHMO results, nanotubes longer than $100 \AA$ would be metallic. However, given the above discussion on the limitations of the computational techniques, the transition to the metallic state likely occurs at longer lengths, probably in the range of $10-20 \mathrm{~nm}$.

\section{CONCLUSIONS}

We have investigated the electronic structure of finite-length armchair carbon nanotubes using a number of abinitio and semi-empirical quantum chemistry techniques. Electrons in carbon nanotubes are confined along their circumference. In this study we imposed an additional confinement along the tube axis by using short tube segments. We found that armchair tubes that are metallic when infinitely long, develop a band-gap. The value of the band-gap decreases with increasing tube length, but the decrease is not monotonic; it shows a well defined oscillation in short tubes. This oscillation can be accounted for in terms of the changes in the bonding characteristics of the HOMO and LUMO orbitals of the tubes as a function of their increasing length. Finite size graphene sheets were also found to have a finite band-gap, but no clear oscillation was observed. The DOS spectrum of short nanotubes evolves with increasing length from that characteristic of a 0-D system to that characteristic of a delocalized 1-D system. This transformation is complete already for tubes about $10 \mathrm{~nm}$ long. The chemical stability of the nanotubes, expressed by the binding energy of a carbon atom, is low for short tubes but increases and saturates at tube lengths $<10 \mathrm{~nm}$.

\footnotetext{
* e-mail: rochefor@cerca.umontreal.ca

$\dagger$ e-mail: Dennis.Salahub@umontreal.ca

‡ e-mail: avouris@us.ibm.com

${ }^{1}$ S. Iijima, Nature, 354 (1991) 56.

2 B.I.Yacobson and R.E. Smalley, Am. Sci. 85 (1997) 324.

${ }^{3}$ M.S. Dresselhaus, G. Dresselhaus and P.C. Eklund, Science of Fullerenes and Carbon Nanotubes (Academic Press, San Diego, 1996).
}

${ }^{4}$ R. Saito, M. Fujita, G. Dresselhaus and M.S. Dresselhaus, Appl. Phys. Lett. 60 (1992) 2204. 
${ }^{5}$ J.W. Mintmire, B.I. Dunlap and C.T. White, Phys. Rev. Lett. 68 (1992) 631.

${ }^{6}$ X. Blase, L.X. Benedict, E.L. Shirley and S.G. Louie Phys. Rev. Lett. 72 (1994) 1878.

${ }^{7}$ J.W.G. Wildöer, L.C. Venema, A.G. Rinzler, R.E. Smalley and C. Dekker, Nature 391 (1998) 59.

${ }^{8}$ T.W. Odom, J.-L. Huang, P. Kim and C.M. Lieber, Nature 391 (1998) 62.

${ }^{9}$ L.C. Venema, J.W.G. Wildöer, H.L.J. Temminck Tuinstra, C. Dekker, A.G. Rinzler and R.E. Smalley, Appl. Phys. Lett. 71 (1997) 2629.

${ }^{10}$ S.J. Tans, M.H. Devoret and C. Dekker, Nature 386 (1997) 474.

${ }^{11}$ M. Bockrath, D.H. Cobden and R.E. Smalley, Science 275 (1997) 1922.

12 J. Roncali, Chem. Rev. 97 (1997) 173.

${ }^{13}$ M.W. Schmidt, K.K. Baldridge, J.A. Boatz, S.T. Elbert, M.S. Gordon, J.H. Jensen, S. Koseki, N. Matsunaga, K.A. Nguyen, S.J. Su, T.L. Windus, M. Dupuis and J.A. Montgomery J. Comput. Chem. 14 (1993) 1347-1363.

14 A. St-Amant and Salahub, D.R. Chem.Phys.Lett. 169 (1990) 387.

15 A. St-Amant, Ph.D. Thesis, Université de Montréal, 1992.

${ }^{16}$ deMon-KS version 3.4, M.E. Casida, C. Daul, A. Goursot, A. Koester, L. Pettersson, E. Proynov, A. St-Amant, D.R. Salahub, H. Duarte, N. Godbout, J. Guan, C. Jamorski, M. Leboeuf, V. Malkin, O. Malkina, F. Sim, and A. Vela, deMon Software, 1996.

17 S. Huzinaga and J. Andzelm, Gaussian basis sets for molecular calculations, Physical sciences data; Vol.16, Amsterdam ; New York : Elsevier, 1984.

18 J.P. Perdew and Y. Wang, Phys. Rev. B 33 (1986) 8800.

19 J.P. Perdew and Y. Wang, Phys.Rev. B 46 (1992) 12947.

${ }^{20}$ M.J.S. Dewar and W. Thiel J. Am. Chem. Soc. 99 (1977) 4899-4907.

21 J.J.P. Stewart J. Comput. Chem. 10 (1989) 209-220.

${ }^{22}$ G. Landrum, YAeHMOP (Yet Another Extended Hückel Molecular Orbital Package, Cornell University, Ithaca, NY, 1995).

${ }^{23}$ Gmelin, Handbuch der Anorganishen Chemie, 8th ed., Verlag Chemie, Weinheim, 1968, Vol.14B/2, p.413.

${ }^{24}$ K. Yoshizawa, K. Yahara, K. Tanaka and T. Yamabe, J. Phys. Chem. 102 (1998) 498.

${ }^{25}$ N.W. Ashcroft and N.D. Mermin, Solid States Physics Saunders College Publishing, Philadelphia, (1976).

${ }^{26}$ The EHMO results obtained for the $96 \AA$ nanotube model are included in a previous publication submitted to Chem. Phys. Lett. by the authors. 
${ }^{27}$ W.J. Hunt and W.A. Goddard III, Chem. Phys. Lett. 3 (1969) 414.

${ }^{28}$ M. Kertész, Adv. Quantum Chem. 15 (1982) 161.

${ }^{29}$ U. Salzner, P.G. Pickup, R.A. Poirier and J.B. Lagowski, J. Phys. Chem. 102 (1998) 2572.

${ }^{30}$ D. Bakowies and W. Thiel Chem. Phys. Lett. 192 (1992) 236-242.

${ }^{31}$ M.J.S. Dewar, H.S. Rzepa, J. Am. Chem. Soc. 100 (1978) 784.

${ }^{32}$ W. Thiel Tetrahedron 44 (1988) 7393.

${ }^{33}$ Y.-S. Lee, M. Kertész, J. Chem. Phys. 88 (1988) 2609.

${ }^{34}$ D. Bakowies and W. Thiel J. Am. Chem. Soc. 113 (1991) 3704-3714.

${ }^{35}$ R.M. Dreizler and E.K.U. Gross, Density Functional Theory, Springler-Verlag, Berlin, 1990.

${ }^{36}$ U. Salzner, J.B. Lagowski, P.G. Pickup and R.A. Poirier, J. Comput. Chem. 18 (1997) 1943. 
FIG. 1. Variation of the band-gap of a $(6,6)$ nanotube as a function of its length determined using different computational techniques. The inset gives an expanded view of the band-gap behavior of very short tubes.

FIG. 2. Qualitative description of HOMO and LUMO orbitals as a function of the nanotube length. The black and white circles indicate opposite phases of the molecular wavefunction.

FIG. 3. Molecular orbital diagram for ten orbitals with energies near the band-gap as determined using EHMO.

FIG. 4. Comparison of the band-gap variation for finite and periodic graphene sheet with respect to the $(6,6)$ nanotube structure. The "finite" sheet is the unwrapped structure of the $(6,6)$ nanotube where broken C-C bonds were saturated with hydrogen.

FIG. 5. Influence of the nanotube diameter on the band-gap oscillations and comparison with the results obtained by Yoshizawa et al 24 for an equivalent periodic 2D graphene sheet .

FIG. 6. Variation of the carbon atom binding energy as a function of the length of the growing $(6,6)$ nanotube.

FIG. 7. Electronic structure of a growing $(6,6)$ carbon nanotube obtained using the EHMO technique. Energy resolution $(\omega)=0.2 \mathrm{eV}$

FIG. 8. Total (DOS) and local density of states (LDOS) diagrams of a $96 \AA$ armchair $(6,6)$ nanotube (resolution $=0.2 \mathrm{eV})$. The indices in the LDOS diagram give the relative position of the carbon atoms in the nanotube structure (1: boundary, 40: middle of the nanotube) The inset gives an expanded view of the DOS near the Fermi level (E=0 eV). The zero of the DOS scale is indicated by the horizontal line, and the energy resolution is $0.05 \mathrm{eV}$. 


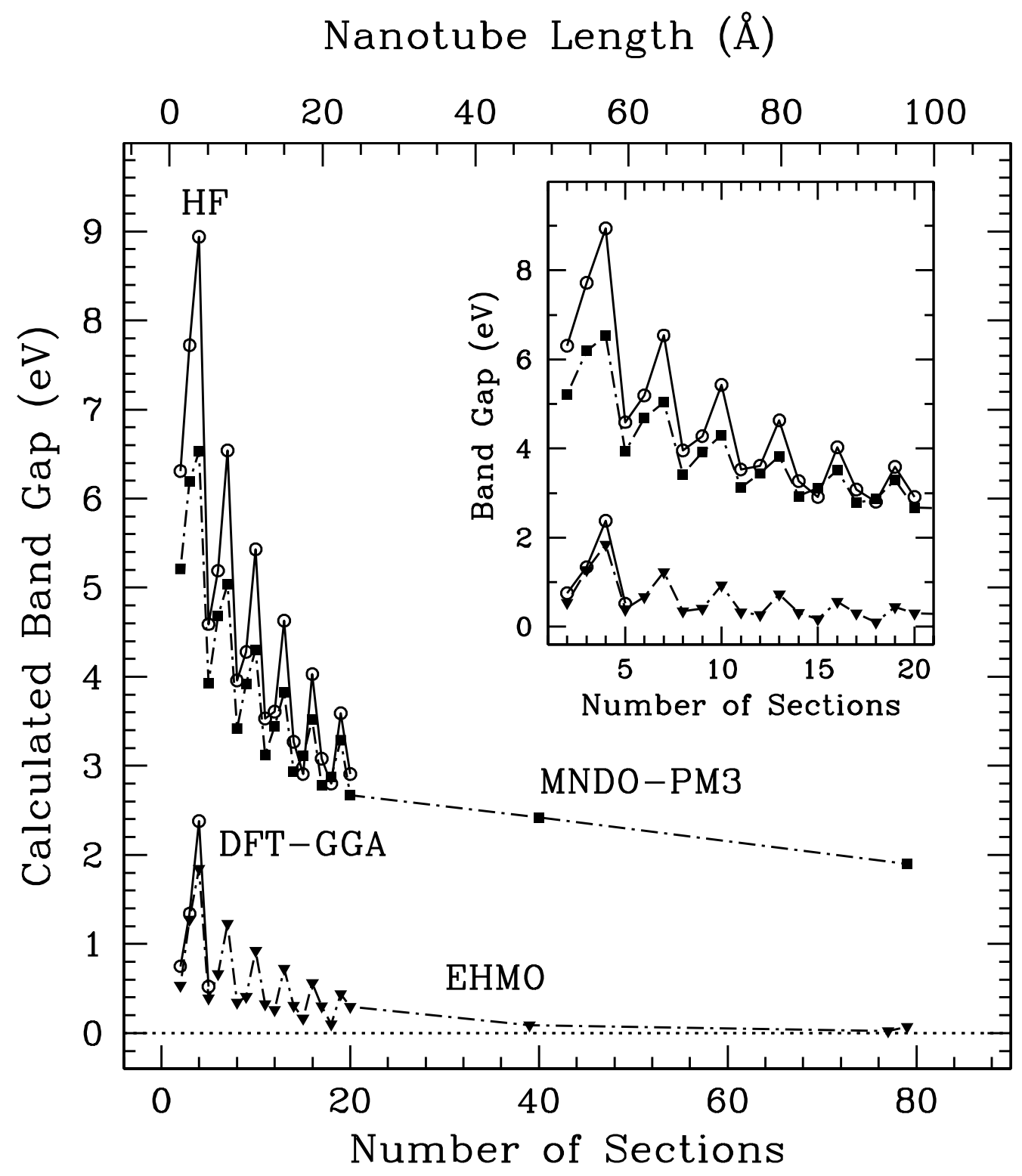

Figure 1. 


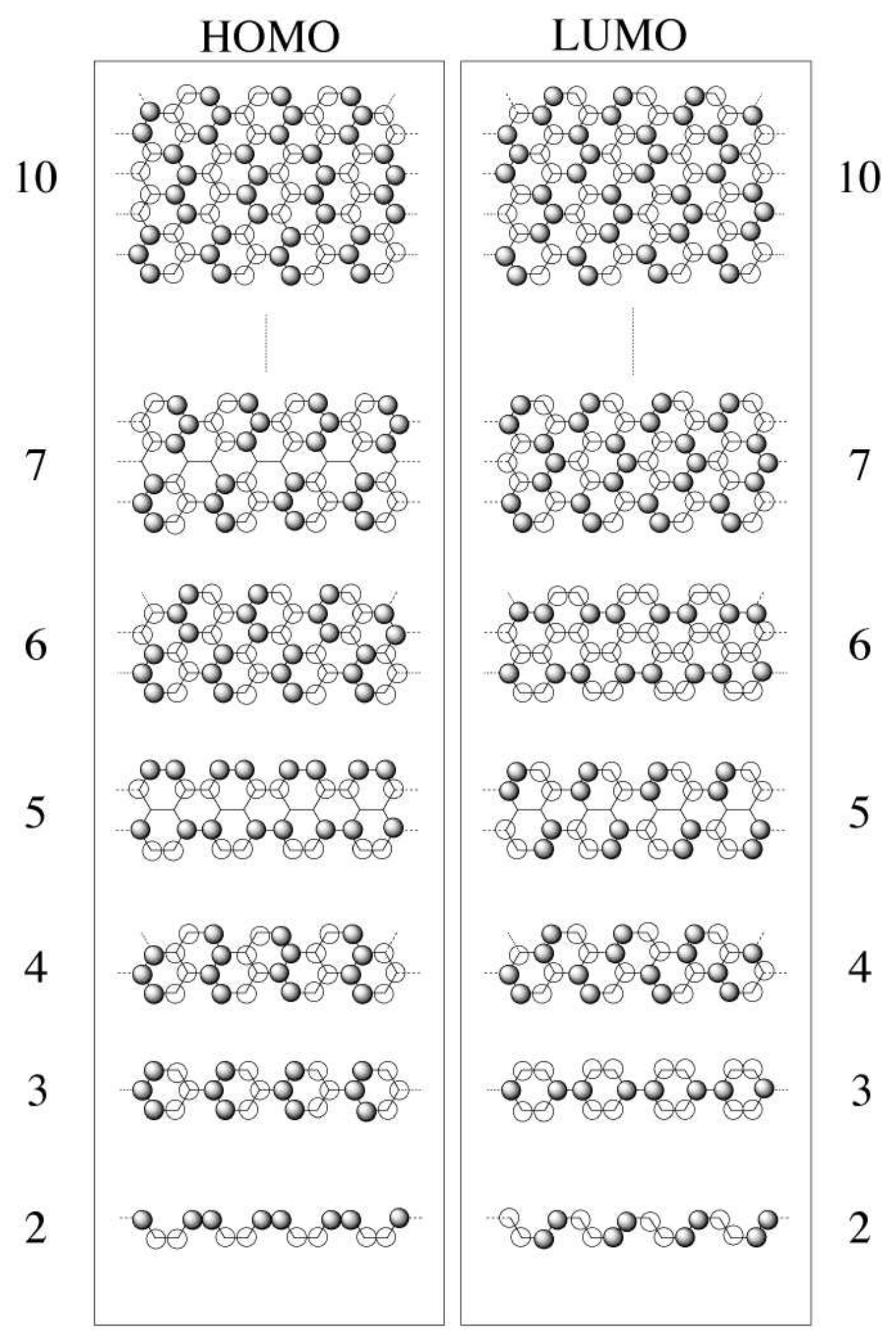

Figure 2 . 


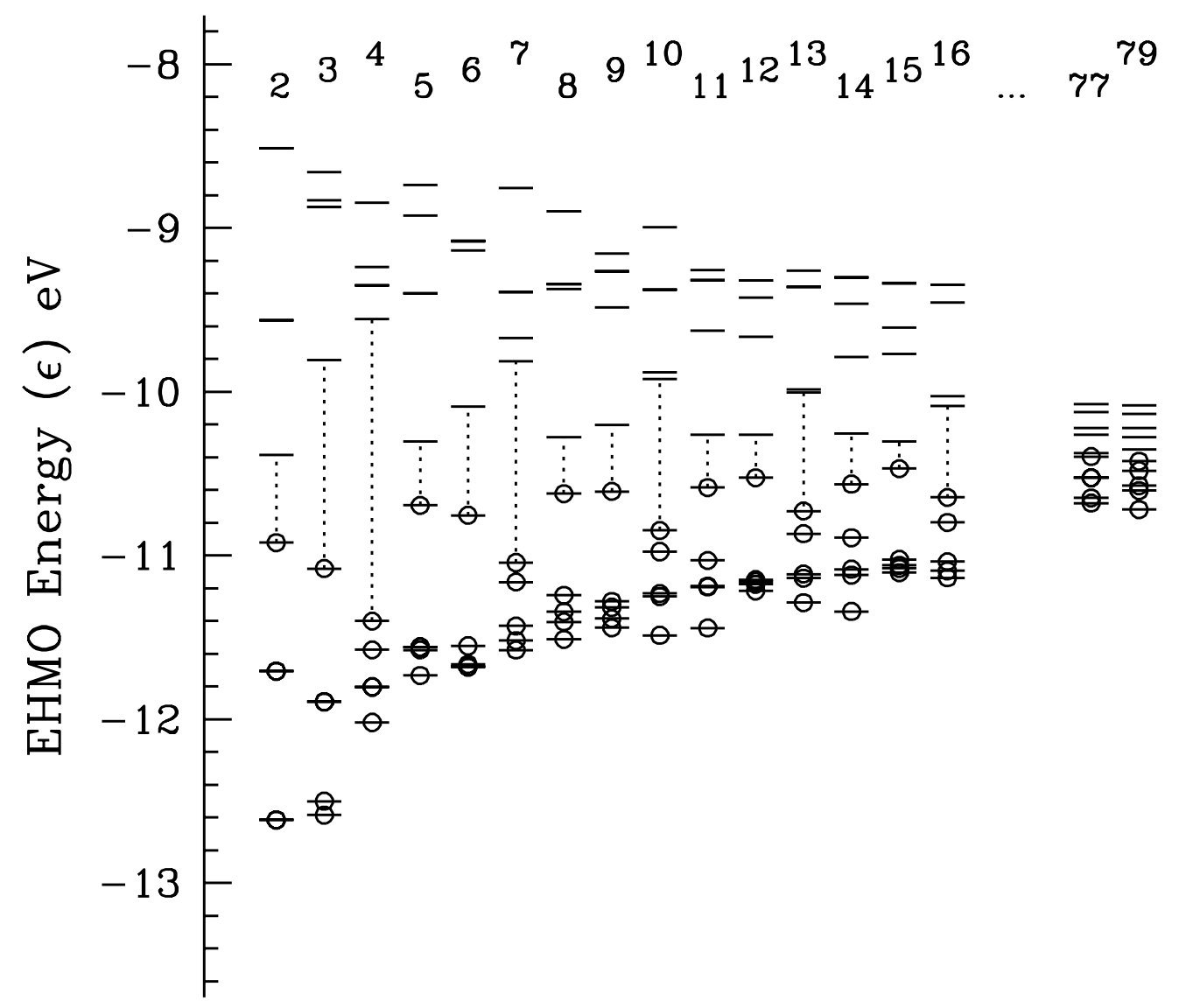

Figure 3. 


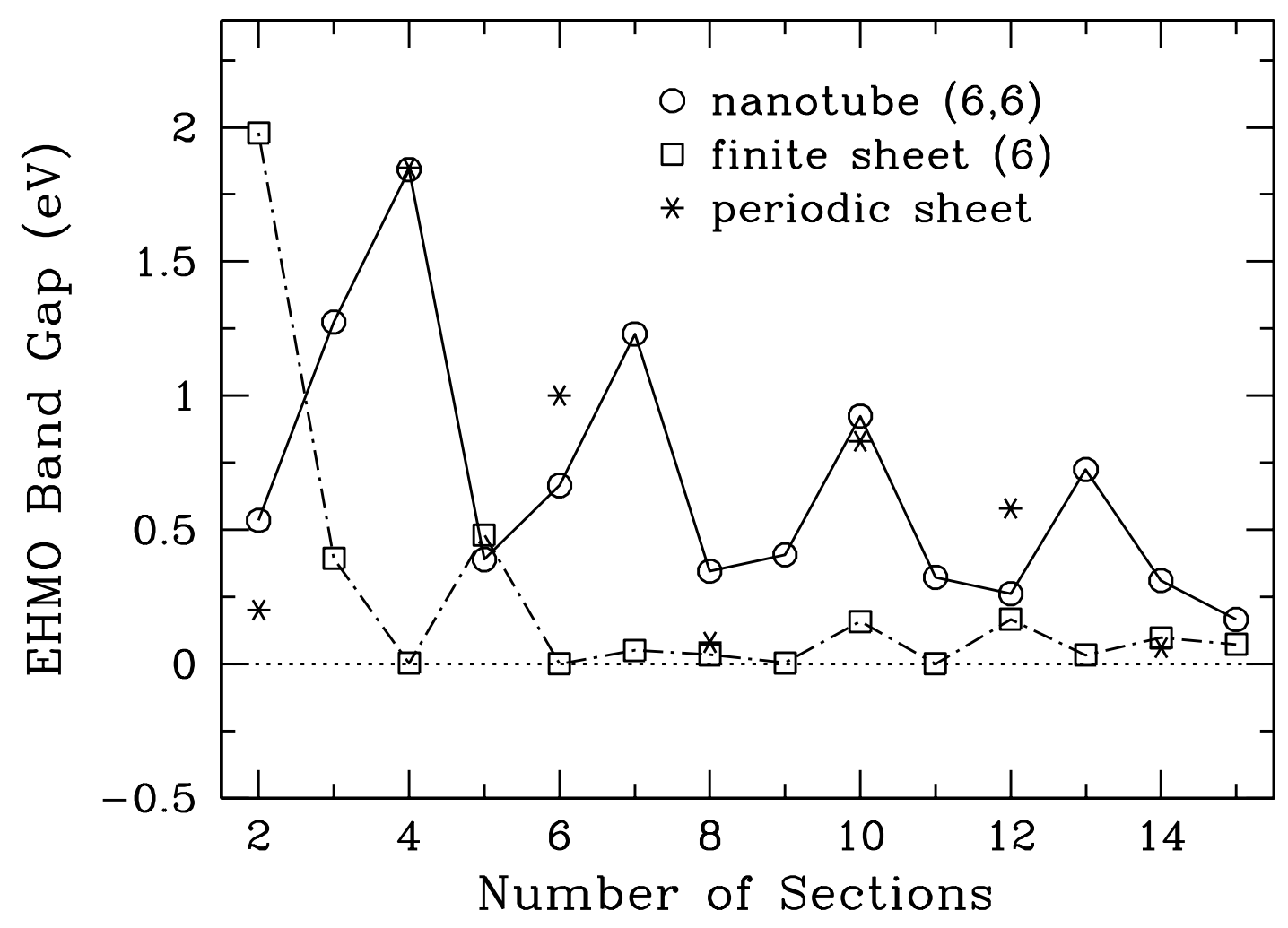

Figure 4 . 


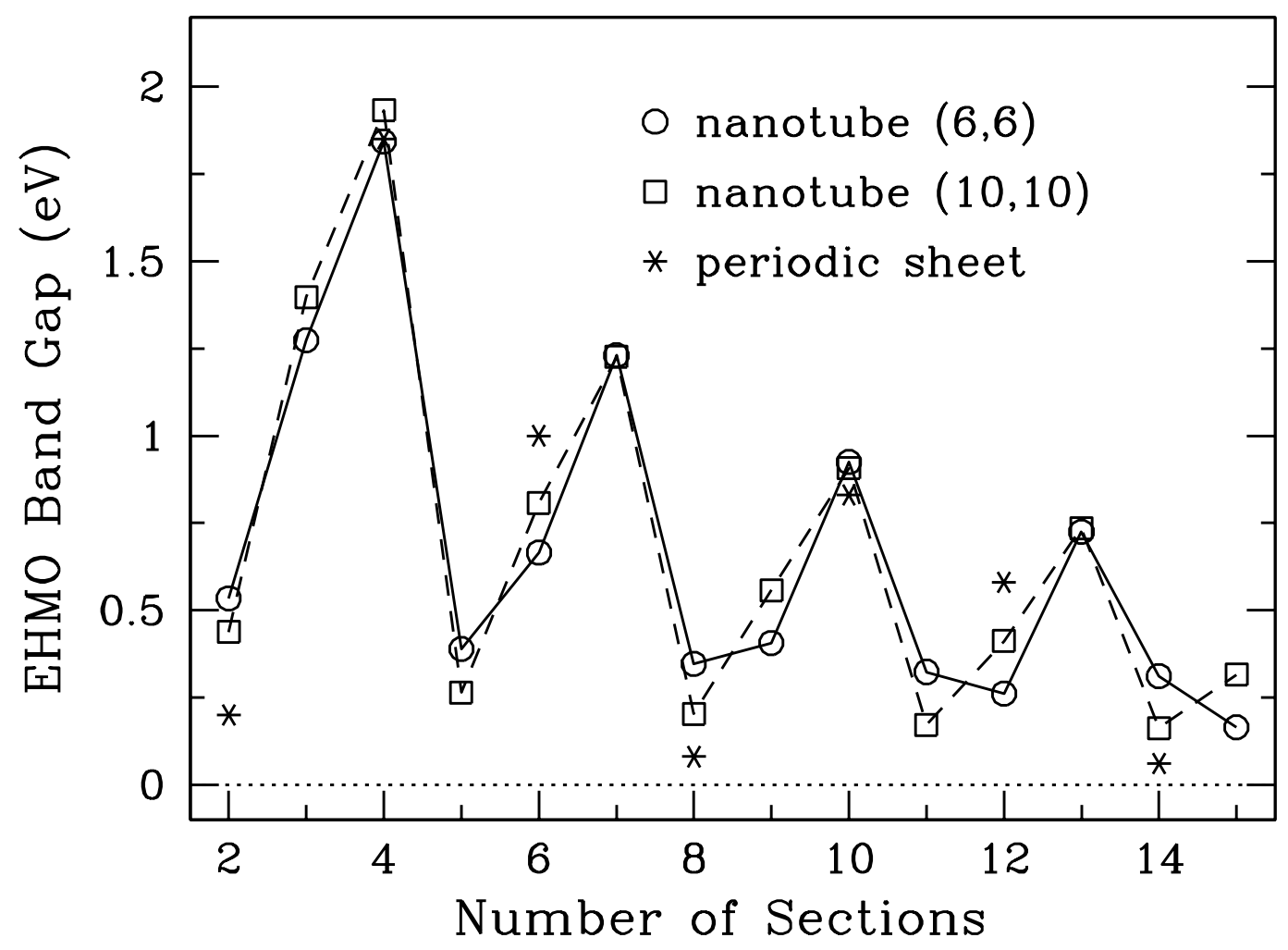

Figure 5 . 


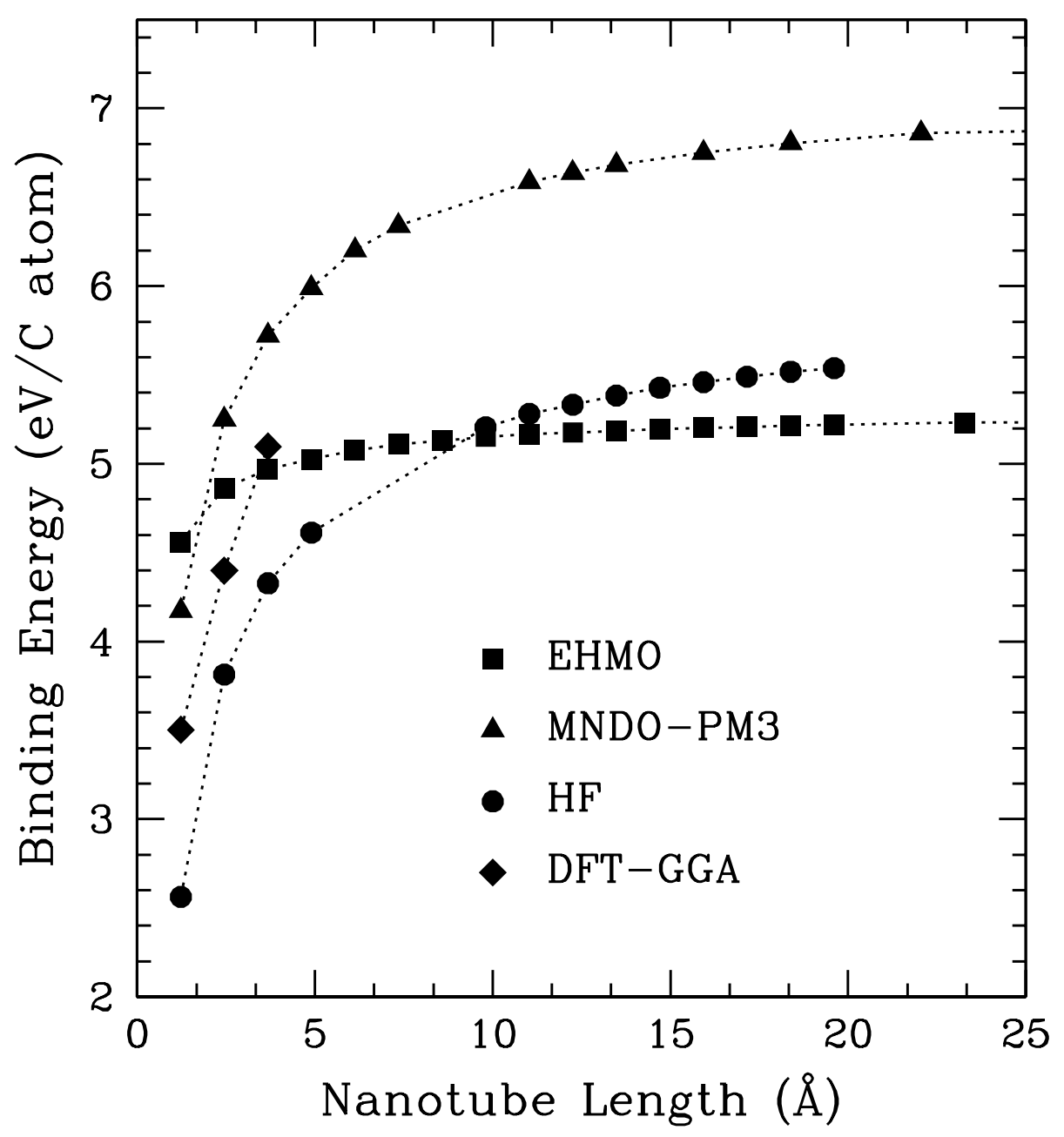

Figure 6 . 


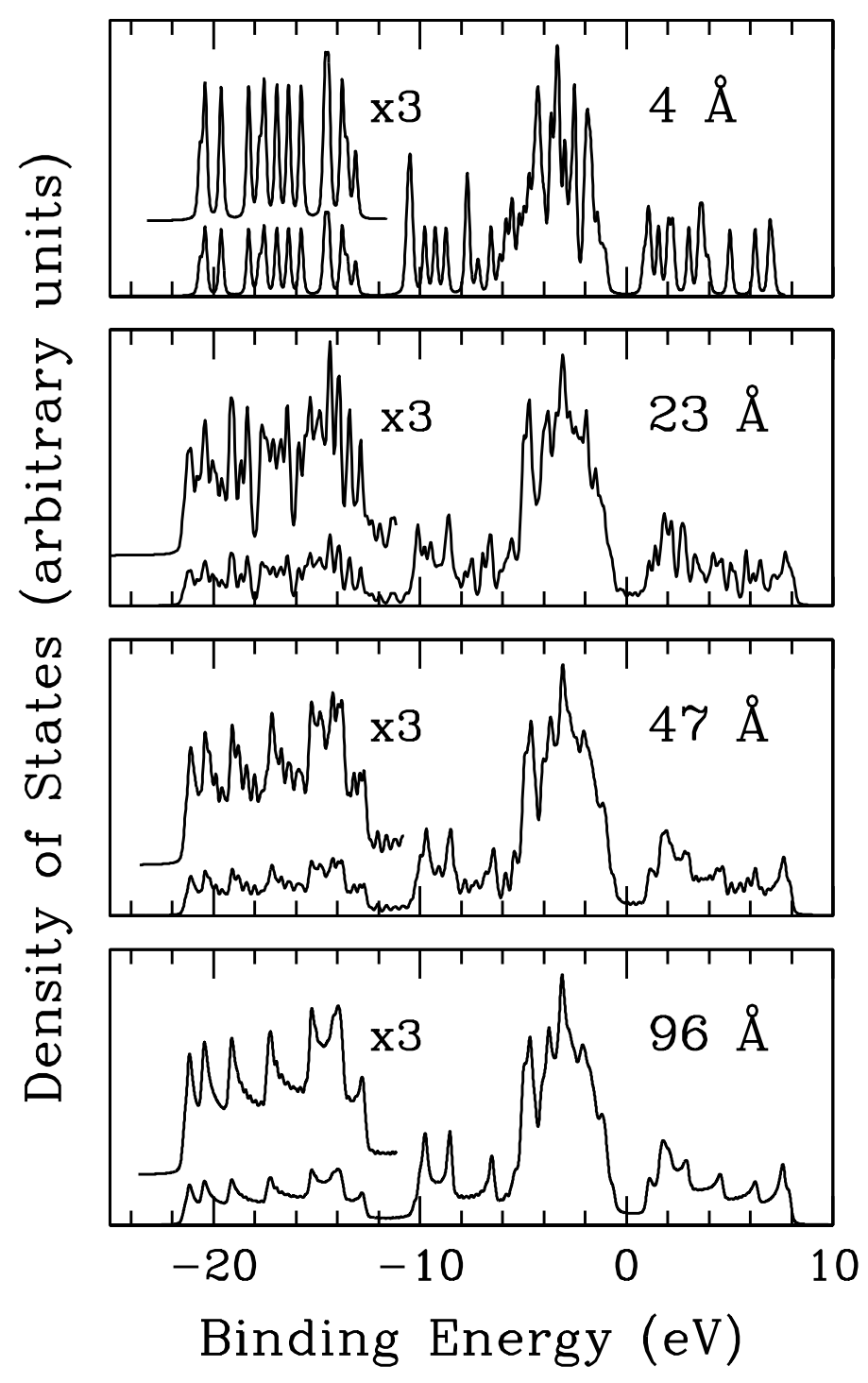

Figure 7. 


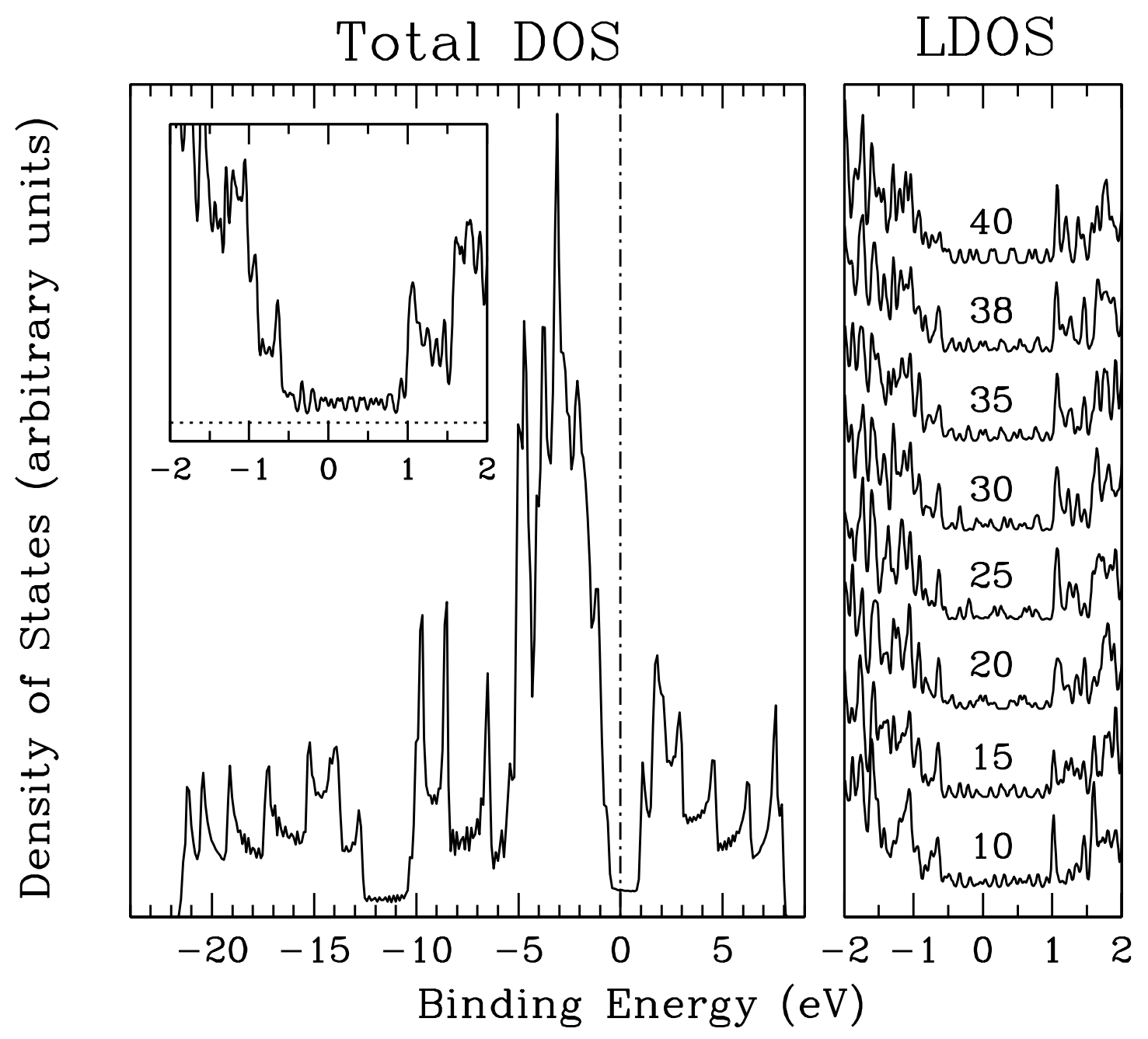

Figure 8 . 